\title{
Distribución y abundancia de nematodos fitoparásitos asociados al cultivo de yerba mate en Itapúa: un riesgo potencial e inminente
}

\author{
Distribution and abundance of plant parasitic nematodes \\ associated with yerba mate in Itapúa: a potential and imminent \\ risk
}

\begin{abstract}
Gabriela Caballero-Mairesse ${ }^{1} \oplus$, Gloria Resquín-Romero ${ }^{2} \oplus$, Fernando Lugo ${ }^{1} \oplus$, Alejandro Gini $^{1}{ }^{1}$, Lucas Alcázar ${ }^{1} \oplus$, Dilma Cano ${ }^{3} \odot$, Horacio D. Lopez-Nicora ${ }^{1,4}{ }^{1 *} *{ }^{1}$ Universidad San Carlos. La Clínica Vegetal. Asunción, Paraguay ${ }^{2}$ Universidad Nacional de Asunción. Facultad de Ciencias Agrarias. San Lorenzo, Paraguay ${ }^{3}$ Ministerio de Agricultura y Ganadería (MAG). Dirección de Extensión Agraria (DEAg). San Lorenzo, Paraguay ${ }^{4}$ The Ohio State University. Department of Plant Pathology. Columbus, Ohio U.S.A.*Autor de correspondencia: lopez-nicora.1@ osu.edu.
\end{abstract}

Recibido: 29 abril 2021

Aceptado: 10 setiembre 2021

Recibido en versión modificada: 8 setiembre 2021

Caballero-Mairesse, G., Resquín-Romero, G., Lugo, F., Gini, A., Alcázar, L., Cano, D., López-Nicora, H. D. (2021). Distribución y abundancia de nematodos fitoparásitos asociados al cultivo de yerba mate en Itapúa: un riesgo potencial e inminente. Investigaciones y Estudios - UNA, 12 (2), 11-27. https://doi.org/10.47133/IEUNA2122b

Resumen. La yerba mate (Ilex paraguariensis $\mathrm{St}$. Hil.) en los últimos años se expandió internacionalmente debido al aumento de las exportaciones; con un $65 \%$ de la producción nacional, el departamento de Itapúa se ha convertido en el mayor productor. $\mathrm{Su}$ rentabilidad $\mathrm{y}$ productividad puede ser afectada por organismos como los nematodos, pero hasta el momento en Paraguay no hay reportes científicos de su presencia en este cultivo. Para identificar la presencia de fitonematodos en la yerba mate del departamento de Itapúa se obtuvieron muestras de suelo de los distritos de Tomás Romero Pereira y Carlos Antonio
López, las cuales fueron procesadas en la Clínica Vegetal de la Universidad San Carlos para su posterior identificación a nivel de género y cuantificación. Los datos obtenidos fueron sometidos a estadística descriptiva y el análisis de índices ecológicos: Índice de Dominancia de Simpson y los Índices de Diversidad y Equidad de Shannon. Los nematodos fitoparásitos identificados fueron Criconemella, Helicotylenchus, Meloidogyne, Pratylenchus, Scutellonema, Tylenchorhynchus, Trichodorus, Xiphidorus y nematodos del grupo Tylenchidos. Además, fueron identificados nematodos benéficos como Aphelenchoides, Predadores, Bacteriófagos y Doriláimidos. Por otra parte, Helicotylenchus, fue el más frecuente, abundante y dominante en las muestras analizadas, seguido por Meloidogyne y Scutellonema. La comunidad de nematodos de las localidades estudiadas fue poco diversa $y$ 
relativamente uniforme. Esta investigación es el primer reporte de nematodos asociados al cultivo de yerba mate en el Paraguay y un llamado de atención a mantenernos alertas para reducir el riesgo potencial que estos presentan a la producción de yerba mate

Palabras clave. yerba mate, Helicotylenchus, Meloidogyne, frecuencia, abundancia, índices ecológicos

Abstract. Yerba mate (Ilex paraguariensis St. Hil.) has in recent years become a culturally and socially important crop for Paraguay. Moreover, it has also stood out economically, prompting an expansion of production at international levels due to increased exports. The department of Itapúa in Paraguay is responsible for $65 \%$ of the national production. Its profitability and productivity can be affected by organisms such as nematodes, but so far in Paraguay there have been no scientific reports of presence of these animals in the crop. To identify the presence of phytonematodes in the yerba mate of the department of Itapúa, soil samples were obtained from the districts of Tomás Romero Pereira and Carlos Antonio López, which were processed in the Plant Clinic of the San Carlos University, for their subsequent taxonomic gender identification and quantification. The data obtained were subjected to descriptive statistics and the analysis of ecological indices: Simpson's Dominance Index and Shannon's Diversity and Equity Index. The phytoparasitic nematodes identified were Criconemella, Helicotylenchus, Meloidogyne, Pratylenchus, Scutellonema, Tylenchorhynchus, Trichodorus, Xiphidorus and nematodes of the Tylenchida group. In addition, beneficial nematodes such as Aphelenchoides, Predators, Bacteriophages and Dorylaimida were identified. On the other hand,
Helicotylenchus was the most frequent, abundant and dominant genus species in the analyzed samples, followed by Meloidogyne and Scutellonema. The nematode community of the studied localities was not very diverse and were relatively uniform. This research is the first report of nematodes associated with yerba mate crops in Paraguay and is a call to attention to reduce the potential risk that these organisms present to the production of yerba mate.

Keywords. yerba mate, Helicotylenchus, Meloidogyne, frequency, abundance, ecological index.

\section{INTRODUCCIÓN}

La yerba mate (Ilex paraguariensis St. Hil.) es una especie arbórea nativa de América del Sur, presentando una amplia dispersión geográfica en Argentina, Brasil y Paraguay, debida a las óptimas condiciones agroecológicas proporcionadas por estas regiones. Dicha extensión es de $540.000 \mathrm{~km}^{2}$, lo cual corresponde a alrededor del $3 \%$ del territorio sudamericano (Oliveira \& Rotta, 1985; Cubas Báez et al., 2018).

Para el Paraguay además de ser cultural y socialmente importante en términos de representatividad y consumo como infusión fría o caliente, económicamente, en los últimos años, su producción ha tenido un incremento exponencial. Dando lugar al aumento de su exportación para abastecer la alta demanda de los productos a base de yerba mate, teniendo que en el 2019 fueron exportados 4.196.196 kg netos (Enciso, 2019). De esta forma, a nivel nacional la superficie plantada en la zafra 2019/2020 fue de 26.215 ha, 4 \% más comparada a la zafra 2018/2019. La producción total fue de 132.739 t, experimentando un aumento de 
5,3\% en comparación a la zafra anterior y también, su rendimiento presentó un aumento de $1,3 \%$. Por su parte, sobresale el departamento de Itapúa por su productividad, siendo responsable del $65 \%$ de la producción nacional, cubriendo una superficie de 15.640 ha (Maciel et al., 2020).

Naturalmente este cultivo se desenvolvía en áreas boscosas, lo que en la actualidad ha cambiado a causa de su domesticación para la producción en sistemas de monocultivo, resultando en un aumento del área cultivada, lo que consecuentemente, produce una reducción del espaciamiento entre plantas. Esta situación propicia el desarrollo y diseminación de patógenos que afectan negativamente el estado fitosanitario de estas plantas, tanto en las etapas de producción de mudas en viveros como en el campo (Grigoletti Junior \& Auer, 1996; Grigoletti Junior et al., 2000).

Los nematodos fitoparásitos se encuentran entre los patógenos que pueden afectar negativamente la productividad y rentabilidad de diversos cultivos, según Abad et al. (2008) causan una pérdida anual global de 157 mil millones de dólares y el más destacado por los graves daños ocasionados es Meloidogyne. En este contexto, en el caso de la yerba mate Lozano y Santos (1991), resaltan que el nematodo de la agalla (M. incognita) es responsable de pérdidas económicas de $56 \%$ en áreas de producción de este cultivo. Por otra parte, M. incognita produjo una reducción de $63 \%$ de la biomasa de la parte aérea de la yerba mate, como así también una disminución de $53 \%$ de la biomasa de la raíz. En cuanto que $M$. paranaensis redujo en un $53 \%$ y $6 \%$ la biomasa de la parte aérea y raíz, respectivamente (Santiago et al., 2000). Adicionalmente, la ocurrencia de individuos del género Meloidogyne, además de limitar el desarrollo del cultivo, puede favorecer la entrada de patógenos causantes de enfermedades radiculares.

De acuerdo a lo expuesto por Grigoletti Junior \& Auer (1996), el control y manejo fitosanitario es fundamental, principalmente en la fase inicial del cultivo durante la producción de mudas, ya que el estrés por exceder su tiempo de permanencia bajo invernadero, puede predisponer la susceptibilidad al ataque de nematodos.

Por consiguiente, es necesario tomar medidas para el manejo con el fin de evitar futuras epidemias y mitigar perjuicios en los cultivos. El manejo integrado de nematodos es la mejor forma de prevenir el aumento de poblaciones de estos organismos, debido a que por la combinación de diferentes métodos se puede frenar a tiempo los efectos dañinos de los fitonematodos (Mesa-Valle et al., 2020).

Por otro lado, es fundamental identificar la comunidad establecida en un área determinada, para obtener información de forma simplificada por medio de índices como la diversidad, riqueza y distribución de géneros y especies, pues estos índices expresan en valores numéricos la complejidad de una comunidad de individuos (Neher \& Darby, 2009; Semensatto Junior, 2003).

El éxito de la calidad de la producción está extremadamente ligado a la sanidad del cultivo durante todo su ciclo productivo, lo cual radica evidentemente en su importancia económica y social (IICA et al., 2019). Pese a esto, el escenario de la investigación científica y recolección de datos de los problemas fitosanitarios de la yerba mate a nivel nacional e internacional aún es limitado, motivando a generar información de relevancia acerca de la presencia y cantidad de estos nematodos y así, realizar estudios que puedan auxiliar y orientar a los 
productores dedicados a pequeñas y grandes superficies de plantaciones.

Por lo anteriormente expuesto, los objetivos de este trabajo fueron determinar la presencia, abundancia y distribución de nematodos fitoparásitos en cultivos de yerba en el departamento de Itapúa. Como también, determinar los parámetros ecológicos de la comunidad de nematodos mediante el índice de dominancia de Simpson y los índices de diversidad y equidad de Shannon.

\section{MATERIALES Y MÉTODOS}

Muestreo de suelo. En el mes de octubre de 2020 muestras de suelo fueron colectadas de 48 campos de producción de yerba mate en 11 localidades del distrito de Tomás Romero Pereira y 3 en Carlos Antonio López, ambos pertenecientes al departamento de Itapúa (Figura 1). El muestreo se realizó según la metodología de Coyne et al. (2009), en donde las muestras de suelo fueron colectadas con palas a una profundidad de 20 a $30 \mathrm{~cm}$. Las muestras fueron de tipo compuesta, ya que cada una estuvo constituida por 10 a 20 submuestras tomadas de forma aleatoria, en zigzag y equidistantes. Cada submuestra representó suelo de la rizósfera de un árbol de yerba mate. Una vez colectadas, las muestras se colocaron en bolsas de plástico, identificadas con rótulos que incluían toda la información necesaria sobre las mismas, incluyendo las coordenadas geográficas del campo muestreado, y trasladadas en conservadoras hasta el laboratorio donde se almacenaron en el refrigerador a $4{ }^{\circ} \mathrm{C}$ hasta el momento de ser procesadas.

Extracción, identificación y cuantificación de nematodos. El procesamiento y análisis de las muestras fue realizado en el periodo comprendido de octubre a diciembre de 2020 en el laboratorio de la Clínica Vegetal de la Universidad San Carlos (USC), localizado en la ciudad de Asunción.

Cada muestra de suelo fue homogeneizada y los nematodos fueron extraídos a partir de $100 \mathrm{~cm}^{3}$ de suelo, según el método de extracción flotacióncentrifugación adaptado de Jenkins (1964) y el método del embudo de Baermann (Baermann, 1917).

La cuantificación e identificación de los nematodos a nivel de género fue realizada mediante el microscopio invertido (Olympus CKX53, Tokio, Japón), observados a 40× de magnificación.

Análisis de los datos y elaboración de mapas. Los datos referentes a la cantidad máxima, mínima de nematodos por género y su abundancia fueron obtenidos mediante el empleo de estadística descriptiva.

Además, fue obtenida la frecuencia de ocurrencia (FO) de cada uno de los géneros y grupos de nematodos identificados, calculada por la fórmula de Norton (1978):

$\mathrm{FO}=\left(\mathrm{N}^{\mathrm{o}}\right.$ de muestras que contienen un género de nematodo en particular $/ \mathrm{N}^{\circ}$ total de muestras) $\times 100$

Por otro lado, la comunidad de nematodos de las localidades estudiadas fue analizada mediante 


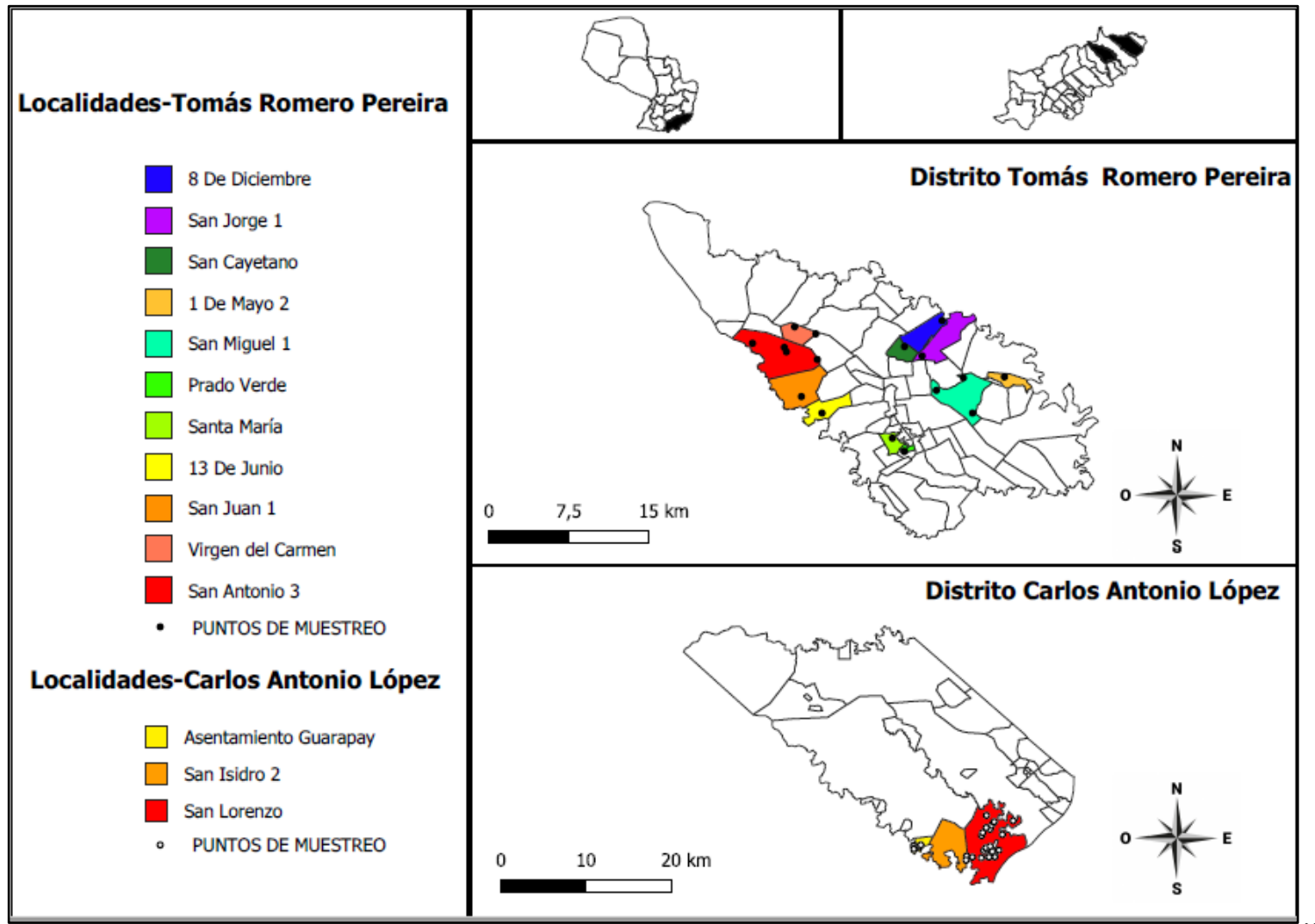

Figura 1. Mapa de los puntos de muestreo de campos de yerba mate de las localidades de los distritos de Tomás Romero Pereira y Carlos Antonio López, departamento de Itapúa, Paraguay.

parámetros ecológicos como, el índice de Dominancia de Simpson (Ds), que mide la probabilidad de que dos individuos, seleccionados al azar, pertenecen a la misma especie y aquellos encontrados en mayor abundancia poseen pesos mayores. Varía de 0 a 1, cuanto más elevado, mayor dominancia (Magurran, 2004; Uramoto, 2005). Dicho índice fue obtenido a través de la siguiente fórmula (Simpson, 1949):

$$
\text { Ds }=\Sigma\left(p_{i}\right)^{2}
$$

donde $p_{i}$ es el \% del taxón i en la población total.

El índice de diversidad (H') de Shannon-Wiener, el cual atribuye mayor peso a especies raras y abundantes. El rango de diversidad es de 0-4, valores próximos a 0 son considerados bajos, valores por encima de 2,5 se consideran altos. Cuanto mayor es el índice H', mayor es la diversidad en el área considerada (Castellón, 2009; Uramoto et al., 2005). Este índice está dado mediante la fórmula (Shannon \& Weaver 1963):

$$
\mathrm{H}^{\prime}=-\Sigma p_{i} \times \ln \left(p_{i}\right)
$$

donde ln es el logaritmo natural.

El índice de equidad (J') de Shannon (Shannon y Weaver 1949) el cual expresa la uniformidad entre las abundancias de diferentes grupos taxonómicos de la comunidad. El índice J' varía de 0 a 1, cuanto mayor es su valor, mayor es la equidad de los géneros de nematodos en el área estudiada (Semensatto Junior, 2003). El índice J' se obtuvo mediante la siguiente fórmula:

$$
\mathrm{J}^{\prime}=\mathrm{H}^{\prime} / \log (S)
$$

donde $S$ es el número total de géneros.

Para la elaboración de los mapas se utilizó el programa QGIS versión 3.18 en el cual después de localizar los puntos de muestreo se procedió a la 
descarga, en formato shapefile, de los departamentos, distritos y localidades de Itapúa del sitio web del Instituto Nacional de Estadística (INE). Fue utilizada la función convex hull, para realizar las interpolaciones, con sus respectivos buffers, mediante el método IDW (Inverse Distance Weighted), y se exportó como ráster.

\section{RESULTADOS}

Frecuencia de ocurrencia de los géneros de nematodos identificados. En las muestras analizadas fueron identificados 8 géneros de nematodos fitoparásitos, correspondientes a Criconemella, Helicotylenchus, Meloidogyne, Pratylenchus, Scutellonema, Tylenchorhynchus, Trichodorus, Xiphidorus y, además, nematodos del grupo Tylenchidos.

La mayor frecuencia de ocurrencia registrada correspondió al género Helicotylenchus con un 100\%; seguido de Meloidogyne, Scutellonema y el grupo Tylenchidos con $54 \%, 50 \%$ y $31 \%$, respectivamente. Por el contrario, los géneros menos frecuentes fueron Xiphidorus, Trichodorus, Pratylenchus, Criconemella y Tylenchorhynchus con frecuencias de 17 a $2 \%$. Los nematodos de vida libre más frecuentes fueron los Doriláimidos con $96 \%$, siguiéndoles los Bacteriófagos (90 \%),

Tabla 1. Estadística descriptiva y frecuencia de ocurrencia de los nematodos fitoparásitos y benéficos identificados en los distritos de Tomás Romero Pereira y Carlos Antonio López, departamento de Itapúa, Paraguay.

\begin{tabular}{rcccc}
\hline Géneros y grupos de nematodos & Mínimo & Máximo & Media* & $\begin{array}{c}\text { Frecuencia de } \\
\text { ocurrencia }(\%) *\end{array}$ \\
\hline Helicotylenchus & 12 & 332 & 107 & 100 \\
Meloidogyne & 0 & 80 & 9 & 54 \\
Scutellonema & 0 & 194 & 18 & 50 \\
\hline
\end{tabular}


Continúa Tabla 1

\begin{tabular}{rcccc}
\hline Géneros y grupos de nematodos & Mínimo & Máximo & Media* & $\begin{array}{c}\text { Frecuencia de } \\
\text { ocurrencia (\%)* }\end{array}$ \\
\hline Tylenchidos & 0 & 20 & 1 & 31 \\
Xiphidorus & 0 & 26 & 1 & 17 \\
Trichodorus & 0 & 4 & 0,2 & 6 \\
Pratylenchus & 0 & 2 & 0,2 & 8 \\
Criconemella & 0 & 6 & 0,2 & 4 \\
Tylenchorhynchus & 0 & 4 & 0,1 & 2 \\
Doriláimidos & 0 & 54 & 17 & 96 \\
Bacteriófagos & 0 & 46 & 17 & 69 \\
Predadores & 0 & 22 & 4 & 67 \\
Aphelenchoides & 0 & 20 & 4 & 90 \\
\hline
\end{tabular}

*Individuos $/ 100 \mathrm{~cm}^{3}$ de suelo

Índices ecológicos. Índice de Dominancia de

Simpson. Para este análisis fueron tenidos en cuenta los géneros de nematodos fitoparásitos más predominantes, siendo estos Helicotylenchus, Meloidogyne y Scutellonema como así también, los nematodos benéficos identificados en las localidades estudiadas. De esta manera, considerando que los valores de este índice presentan un rango de 0 a 1 , resalta el género Helicotylenchus con una dominancia de 0,88 a 0,4 en más de $71 \%$ de las localidades en estudio y 0,34 a 0,07 en las restantes. Sin embargo, se observaron valores muy inferiores para Meloidogyne y Scutellonema.

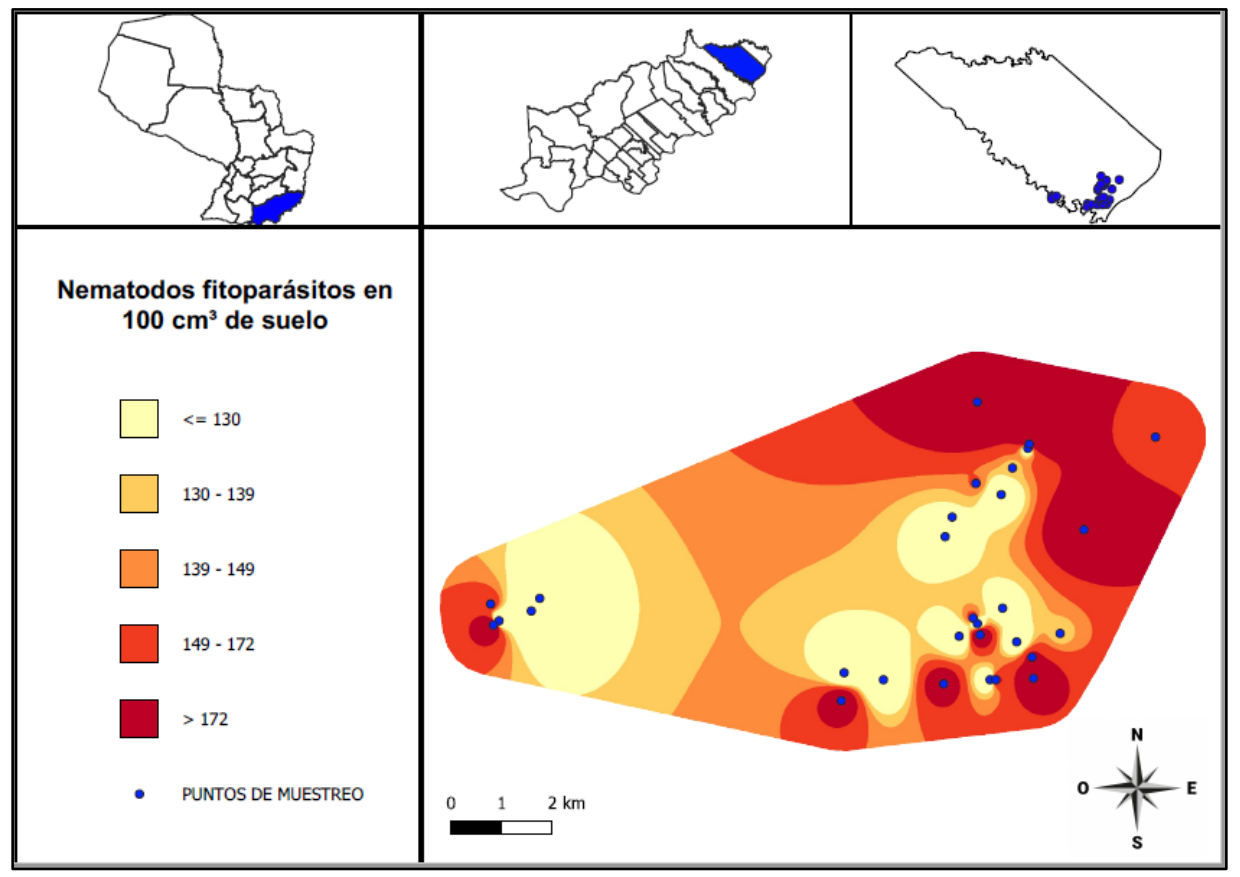

Figura 2. Abundancia de nematodos fitoparásitos asociados al cultivo de yerba mate en el distrito de Carlos Antonio López, Departamento de Itapúa, Paraguay. 


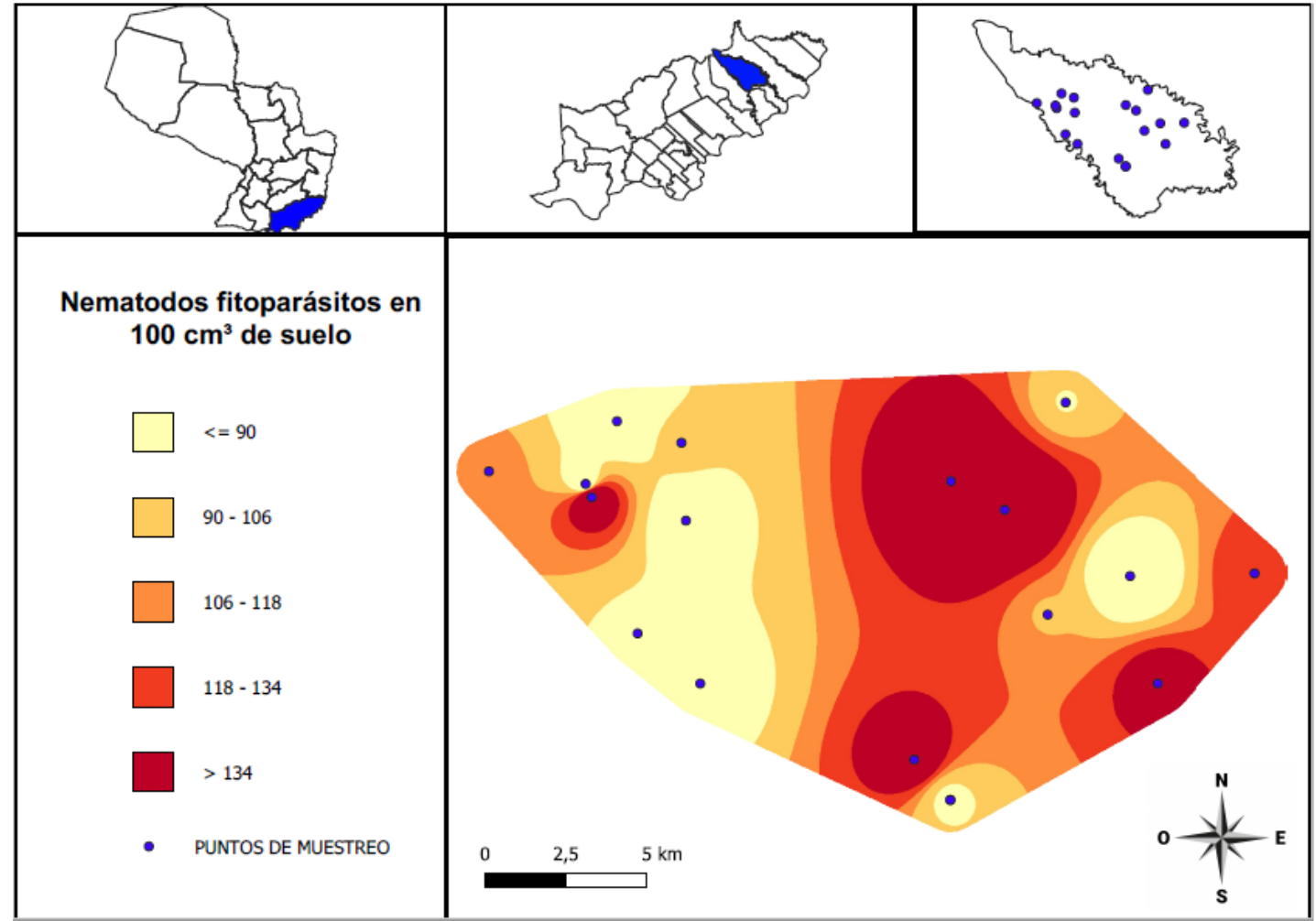

Figura 3. Abundancia de nematodos fitoparásitos asociados al cultivo de yerba mate en el distrito de Tomás Romero Pereira, departamento de Itapúa, Paraguay.

Índice de diversidad de Shannon-Wiener. La localidad que presentó una mayor diversidad frente a las demás fue San Juan 1 con 1,59. A pesar de esto, el rango de referencia de este índice contempla valores de 0 a 4 , siendo 4 el indicador de una alta diversidad, y menor a 2,5 baja diversidad, lo cual indica que en general, los valores obtenidos de 1,59 a 0,4 revelan que la comunidad de nematodos en estas 14 localidades es poco diversa.

Los resultados anteriores se representaron en mapas, en los cuales los puntos en donde hubo una mayor dominancia de unos pocos géneros, la diversidad fue baja, por el contrario, los puntos donde la dominancia de unos pocos géneros fue baja, se observó una alta diversidad (Figura 4 y 5).

Índice de equidad de Shannon. Considerando que el valor máximo del rango de referencia es 1 , los resultados más elevados de equidad se obtuvieron en las localidades de San Juan 1 con 0,82, Prado Verde con 0,76 y 1 de Mayo 2 con 0,72 . No obstante, los valores próximos a 0 correspondieron a Santa María y Asentamiento Guarapay con 0,25 y 0,29.

Dado que este índice define que tan uniforme es la distribución de las abundancias de los individuos de una comunidad, teniendo en cuenta que el $64 \%$ de las localidades presentan valores de 0,50 a 0,82 , se puede afirmar que la distribución es uniforme. 


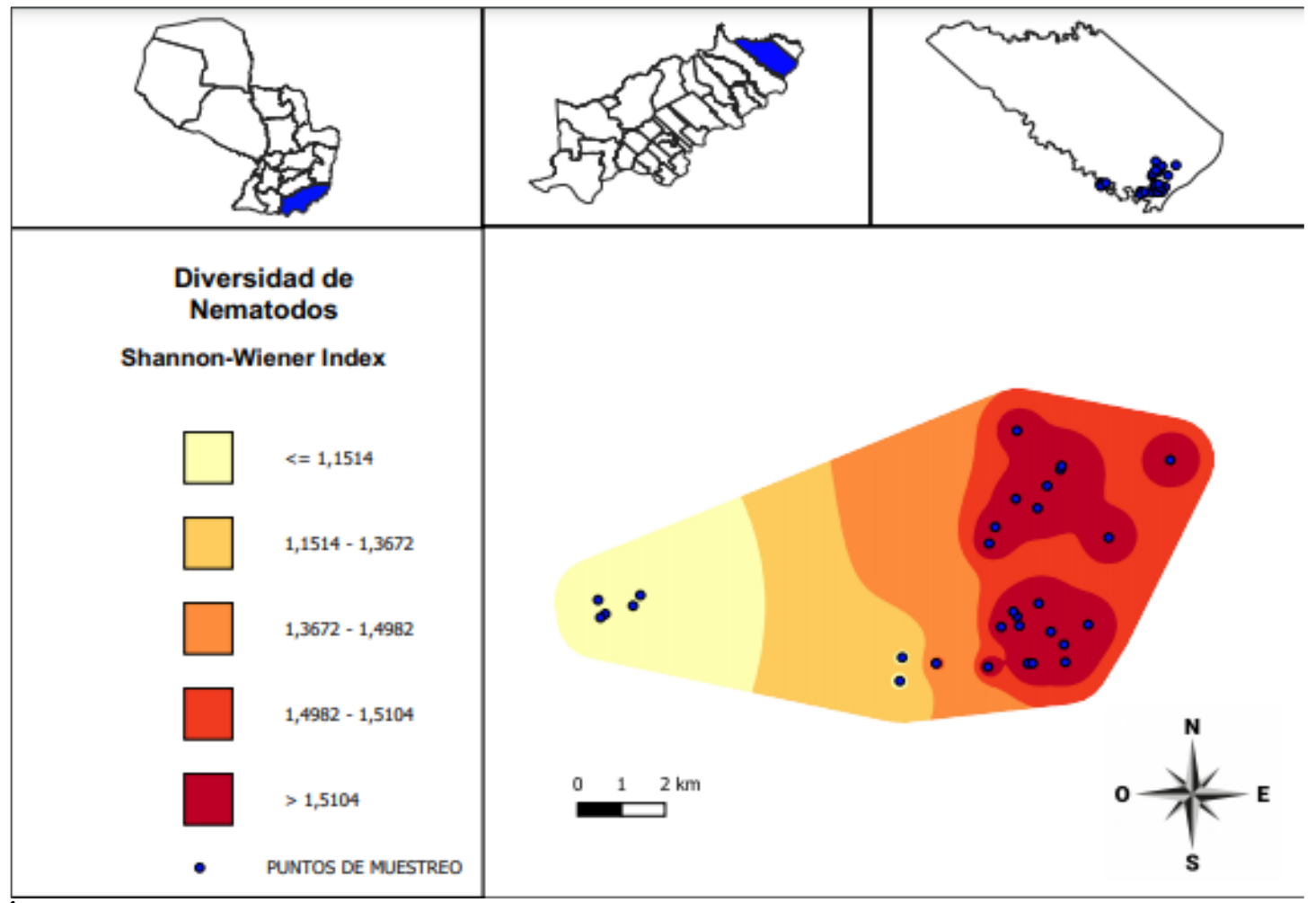

Figura 4. Índice de diversidad de Shannon basado en los nematodos identificados en producciones de yerba mate del distrito en el distrito de Carlos Antonio López, departamento de Itapúa, Paraguay. Interpolación de la abundancia de los nematodos en un contorno de los 30 puntos de muestreo. Las áreas más claras representan una menor diversidad, las áreas más oscuras representan una mayor diversidad.

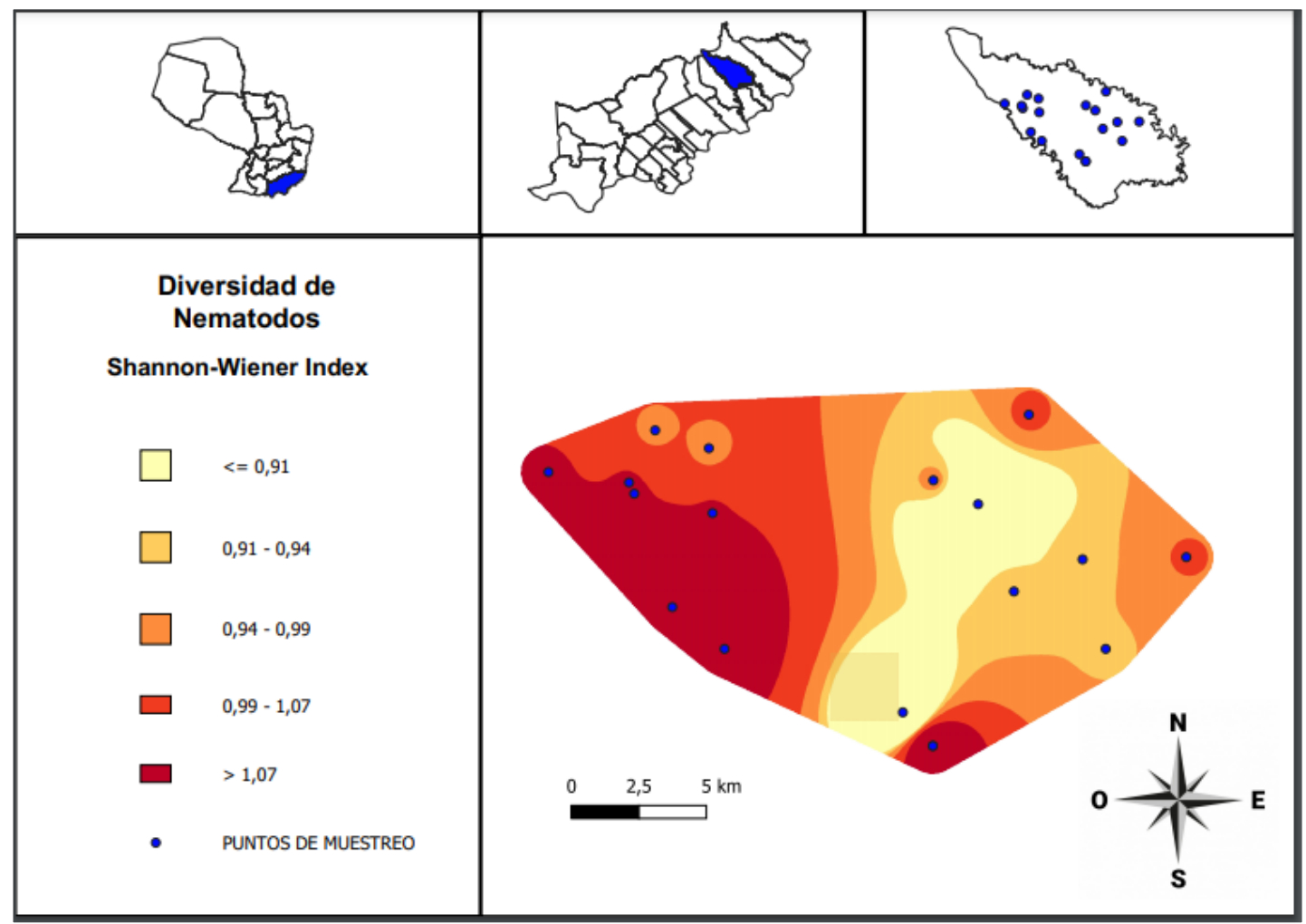

Figura 5. Índice de diversidad de Shannon basado en los nematodos identificados en producciones de yerba mate del distrito de Tomás Romero Pereira, departamento de Itapúa, Paraguay. Interpolación de la abundancia de los nematodos en un contorno de los 18 puntos de muestreo. Las áreas más claras representan una menor diversidad, las áreas más oscuras representan una mayor diversidad. 


\section{DISCUSIÓN}

\section{Frecuencia de ocurrencia y abundancia de} nematodos en $100 \mathrm{~cm}^{3}$ de suelo de los géneros de nematodos identificados. La presencia de nematodos en una producción agrícola puede desencadenar una serie de perjuicios como la reducción de la producción y la calidad de los cultivos que resultan en pérdidas económicas, muchas veces devastadoras para los productores (Jones et al., 2013). Se tienen como opciones varias medidas de mitigación, pero el primer paso para suprimir las poblaciones de estos organismos y así, evitar riesgos y consecuencias es identificando su presencia en los campos como así también, cuantificándolos, pues la cantidad encontrada permitirá tomar una mejor decisión del tipo de manejo (Bogale et al., 2020; Neves et al., 2009).

Este primer paso se ha logrado con este estudio, ya que es el primer reporte de nematodos asociados al cultivo de yerba mate en el Paraguay, en el cual fueron obtenidos datos de la presencia y abundancia de nematodos fitoparásitos que podrían dar indicios de posibles afecciones en producciones de este cultivo.

Teniendo en cuenta el hallazgo de 8 géneros de nematodos en esta investigación, Carvalho et al. (2011) afirman que en áreas cultivadas es habitual encontrar un limitado número de géneros, pero en altas poblaciones. Los monocultivos, como el caso de la yerba mate en el Paraguay, son hospederos ideales para nematodos fitoparásitos, aún más si esta práctica se extiende a lo largo del tiempo (Neher et al., 2019; Dinardo-Miranda, 2006). Lo que explicaría la identificación, en las localidades estudiadas, de nematodos con características fitoparasitarias como Meloidogyne y Pratylenchus.

Entre los nematodos identificados el que precisamente requiere de mayor atención es el nematodo de la agalla, Meloidogyne, ya que se ha llegado a un consenso de que se clasifican entre los fitonematodos más importantes a nivel mundial, pues están dispersos en varios tipos de ambientes, poseen un hábito polífago, tienen una inmensa lista de hospederos susceptibles $y$ en condiciones favorables son altamente prolíferos (Bernard \& Bonsi, 2017). Consecuentemente, provocan pérdidas en los cultivos y el fracaso de la producción, siendo así, considerado como un organismo que confiere alto riesgo para la seguridad alimentaria (Ramos et al., 2019).

Las características propias del endoparásito Meloidogyne le confieren producir un innegable impacto negativo en los cultivos, y en lo que respecta a la yerba mate las investigaciones aún son insuficientes y desactualizadas, de esta forma, Lozano \& Santos (1991) y Santiago et al. (2000) se encuentran entre los pocos reportes disponibles y afirmaron que el nematodo de la agalla produjo daños y redujo el rendimiento del cultivo de yerba mate.

Este nematodo puede infectar y penetrar todo tipo de raíces, desde aquellas superficiales hasta la pivotante más lignificada, a una profundidad de más de $50 \mathrm{~cm}$ (Ferreira et al., 2007). En este sentido, el tipo de raíz pivotante de la yerba mate, con raíces secundarias, que mayormente están en el horizonte superficial del suelo (Burtnik, 2006) le podrían 
conferir susceptibilidad a Meloidogyne.

Lo expuesto anteriormente se verifica en plantas que se agrupan en el mismo género, ya que presentan características similares, por lo que según Horst (2013) las plantas arbustivas y arbóreas del género Ilex como I. aquifolium, I. crenata, I. cornuta e I. opaca son hospederas de varias especies de Meloidogyne. Así, también caracterizada por presentar raíz pivotante I. aquifolium ha demostrado susceptibilidad a $M$. silvestris, revelando altas tasas de infección en sus raíces, además de la presencia de graves agallas (Castillo et al., 2009). Esto evidencia la agresividad y capacidad de Meloidogyne de ocasionar daños en las raíces de plantas perennes con raíces pivotantes, por esta razón puede ser un peligro para plantaciones de yerba mate.

Cabe destacar que Meloidogyne podría infectar las raíces de la yerba mate, pues el café al poseer un sistema radicular similar y ser producido en un sistema de monocultivo, es susceptible a este fitonematodo, siendo considerado el género más peligroso en las áreas de plantaciones de café en Brasil, limitando el rendimiento y destruyendo las raíces (Campos \& Villain, 2005; Campos \& Silva, 2008).

En cambio, el nematodo más frecuente y abundante en $100 \mathrm{~cm}^{3}$ de suelo fue Helicotylenchus, lo que pudo relacionarse a que posee una mejor aptitud biológica que le confiere una mayor sobrevivencia en el suelo, con oportunidades de alimentación superiores a las de otros fitonematodos. Así mismo, en áreas tropicales y sub-tropicales es un nematodo polífago y cosmopolita, que puede desarrollar hábitos alimenticios como ectoparásito, semi-endoparásito o endoparásito de raíces (Luc et al., 2005).

Siguiendo este razonamiento Rocha et al. (2019) destacan que por las características de Helicotylenchus, en el proceso del parasitismo le permiten distribuirse mejor dentro de un área.

Además de esto, su presencia en las áreas cultivadas puede ocasionar daños secundarios, debido a la apertura de puertas de entrada para otros patógenos como hongos y bacterias (Kirsch et al., 2016).

Por otro lado, la considerable frecuencia y abundancia de los nematodos de vida libre en las 48 muestras, podría indicar un buen estado sanitario del suelo de esas zonas productivas, ya que estos son considerados benéficos, pudiendo influenciar en la calidad y nutrición del suelo, viviendo asociados a las raíces sin dañarlas, además de actuar como controladores biológicos (Ritzinger et al., 2010).

Debido a que estos nematodos poseen representantes en la mayor parte de los niveles tróficos del suelo, se puede afirmar que son indicadores potenciales de una amplia variedad de propiedades del suelo (Figueira et al., 2011). Los bacteriófagos están directamente ligados a la descomposición de la materia orgánica, por la ingestión de microorganismos saprófitos, relacionando el aumento de estos individuos con una mayor tasa de descomposición (Ekschmitt et al., 2001).

Así, por utilizar su estilete para penetrar en las hifas de los hongos, las especies micófagas reducen su impacto en los cultivos, propiciando el aumento de la descomposición de la materia orgánica (Goulart, 2007; Almeida \& Andrade, 2010).

La baja frecuencia de algunos nematodos como Pratylenchus, Criconemella, Tylenchorhynchus y Trichodorus, pudo haber ocurrido por la presencia de los predadores, ya que estos se alimentan de organismos habitantes del suelo, reduciendo las 
poblaciones de fitoparásitos, con la capacidad de liberar de nutrientes de forma disponible para las plantas, permitiendo que estas soporten la presencia de fitonematodos en su sistema radicular (Khan \& Kim, 2007).

Índices ecológicos. Según Suárez \& Rosales (2004) una alta presencia de Helicotylenchus en el suelo podría deberse a que es una especie ectoparásita que puede comportarse como un endoparásito migratorio, completando su ciclo en la raíz, posibilitando encontrarlos en mayor cantidad en la rizósfera. Anaya \& Lombo (2008) mencionan que posee un amplio rango de adaptación que le permite ser más tolerante a diferentes cambios físicos y químicos del suelo, lo que resulta en una alta capacidad de incremento poblacional.

Estudios de comunidades de nematodos con especies perennes en sistemas de monocultivo pueden compararse a las plantaciones de yerba mate. De esta forma, los resultados coinciden con Palomares-Rius et al. (2012) que al analizar los suelos y raíces de cultivos de olivo constataron que la mayor predominancia fue de $91 \%$ para Helicotylenchus. De igual manera Meneses et al. (2003) afirman que los sistemas convencionales son afectados por altas poblaciones de nematodos $\mathrm{y}$, por ende, existe una dominancia de unas pocas especies.

Esto concuerda con los resultados obtenidos por Peraza (2010) ya que en un sistema convencional de cultivo de café encontró una menor diversidad de 1,3 en la comunidad de nematodos en comparación a un sistema de bosque natural. Así mismo, constató una equidad de 0,8 ; considerándose relativamente uniforme, debido a que si existen más géneros en una misma finca estos se encuentran equitativamente más distribuidos por lo que será mayor la posibilidad de encontrarlos en futuros muestreos.
De este modo, Arévalo (2014) indicó que la diversidad de nematodos disminuyó de sistemas nativos a sistemas cultivados, de 1,3 a 0,8. Estos cambios se relacionan con la diversidad de plantas y los factores físicos y químicos del suelo (Huang \& Cares, 2006). Por su parte Tomazini et al. (2008) señalaron que la uniformidad presentada en los suelos de bosques y huertos de producción de manzanos fue más alta debido a la alta producción de energía y distribución de recursos.

La acumulación de nematodos en especies perennes está ligada a la producción de biomasa radicular que promueve una mayor actividad biológica en el suelo (Derouard \& Lavelle, 1994). En cambio, que una poca diversidad podría deberse al uso de pesticidas y otros insumos en los sistemas convencionales que reducen la diversidad (Altieri, 1995).

Sin embargo, en especies perennes la diversidad de nematodos parásitos tiende a reducirse. Además, generalmente, la fauna del suelo de producciones agrícolas presenta una menor diversidad y el equilibrio de la comunidad se altera a favor a las especies patógenas (Edwards \& Lofty, 1969; Kandji et al., 2001). Concordando con lo expuesto por Kimenju et al. (2009) quienes encontraron que los nematodos parásitos de plantas eran más abundantes en plantaciones de café y maíz en comparación al bosque natural y las plantaciones forestales.

Las perturbaciones de los suelos a algunos organismos, incluyendo a los nematodos, promueven una mejor adaptabilidad y dominancia en las comunidades, pero consecuentemente la riqueza y diversidad de la fauna del suelo se reducen (Paoletti et al., 1993). Este comportamiento también fue observado por Rivera et al. (2015), ya que sus resultados de diversidad en plantas de arándano 
sugieren que hubo una menor diversidad de géneros de nematodos, y, en consecuencia, una disminución de la riqueza, pero la abundancia por cada género fue elevada, posiblemente debido al sistema de producción anterior.

Las evidencias descritas anteriormente sugieren que la disminución de la diversidad está ligada a una dominancia de unas pocas especies en una comunidad de nematodos, lo cual pudo ser constatado en esta investigación, ya que esta reducción fue directamente proporcional a la presencia de una especie dominante en la región estudiada, así, podemos considerar que los datos obtenidos en uno de los índices pueden dar conocimiento acerca de otros. Por lo tanto, resulta imprescindible, monitorear constantemente parcelas de producción agrícola, complementando esta acción con información de estudios de las poblaciones de nematodos fitoparásitos basados en los índices diversidad y equidad de Shannon y de dominancia de Simpson, los cuales permiten conocer como está distribuida la comunidad con la cual nos deparamos, como también su capacidad de ocasionar daños a los cultivos establecidos en el área productiva, posibilitando la mejor toma de decisiones sobre su manejo (Rocha et al., 2019).

Debido a las bajas poblaciones encontradas se puede elaborar una hipótesis sobre un factor "edad" que puede afectar la dinámica poblaciones de Meloidogyne, pues según Arévalo (2014) en un experimento realizado en un sistema de producción de cacao encontró que las poblaciones de Meloidogyne aumentaban con los años.

Se observó que uno de los géneros menos dominantes fue Pratylenchus con respecto a Meloidogyne, esto pudo haberse debido a que la especie que parasitó primero a las raíces impidió la penetración y desarrollo de la otra, lo que incitaría a un antagonismo entre ambas, este efecto puede deberse al daño que causa en los tejidos la especie que invade primero (Umesh et al., 1994; Fernández \& Ortega, 1982).

Los nematodos de vida libre son sensibles a las perturbaciones, sus bajas poblaciones en los suelos cultivados suelen indicar la degradación de la fertilidad del suelo y de sus propiedades (Bongers \& Bongers, 1998). En cambio, Ovando (2014) destaca que pueden incrementar sus poblaciones ante cualquier perturbación del suelo o bajo condiciones de enriquecimiento de nutrientes siendo una condición que puede interpretarse como indicador de fertilidad del suelo.

En nuestros resultados fue hallado un predominio de los bacteriófagos, observándose una notoria diferencia por parte de Aphelenchoides, lo que concuerda con Neher (1999) que destaca una predominancia de los bacteriófagos sobre los fungívoros, esto puede deberse a que los bacteriófagos ingieren bacterias enteras y tienen pocas defensas. Por lo contrario, los fungívoros deben penetrar las hifas, las cuales poseen cierta resistencia y así, alimentarse del contenido celular, además que ciertos fertilizantes sintéticos pueden frenar la recuperación de los hongos más que de las bacterias (Wardle \& Yeates, 1993; Eno \& Blue, 1954).

Los resultados obtenidos en este estudio son significativos por el hecho de la escasez de informaciones y datos acerca de la ocurrencia de nematodos en cultivos de yerba mate a nivel nacional como internacional. Es necesario realizar más investigaciones que contemplen a todo el departamento de Itapúa con una mejor distribución de los puntos de muestreo, ya que en el caso del 
distrito de Carlos Antonio López dichos puntos estaban concentrados en la región sur del distrito.

Así también, extender estas investigaciones a nivel país, a fin de ampliar el conocimiento de la presencia de los nematodos fitoparásitos en producciones de yerba mate, pues esto sería una herramienta importante en el planeamiento y manejo integrado del cultivo, previendo posibles daños y pérdidas de rendimiento.

\section{CONCLUSIÓN}

Los nematodos fitoparásitos identificados fueron Criconemella, Helicotylenchus, Meloidogyne, Pratylenchus, Scutellonema, Tylenchorhynchus, Trichodorus, Xiphidorus y además, nematodos del grupo Tylenchidos. En cambio, los nematodos de vida libre correspondieron a Aphelenchoides, Predadores, Bacteriófagos y Doriláimidos.

Helicotylenchus, fue el nematodo más frecuente, abundante y dominante en las muestras analizadas, seguido por Meloidogyne y Scutellonema.

La comunidad de nematodos de las localidades de Tomás Romero Pereira y Carlos Antonio López fue poco diversa y relativamente uniforme.

Finalmente, la presencia del nematodo de la agalla (Meloidogyne) en el cultivo de yerba mate representa un potencial peligro, por lo que, actualmente, para definir su parasitismo debemos aún completar los postulados de Koch a fin de conocer e identificar a las especies patógenas encontradas.

\section{AGRADECIMIENTO}

Muy especialmente queremos agradecer a la gerente de la DEAg, MAG de Natalio; a la asociación de yerbateros del Paraguay; a CECTE, Pirapey km 40, Edelira; a los Técnicos de la Municipalidad de Edelira; y a los productores.

Declaración de interés. Los autores declaran no tener conflicto de intereses.

\section{REFERENCIAS BIBLIOGRÁFICAS}

Abad, P., Gouzy, J., Aury, J. M., Castagnone-Sereno, P., Danchin, E. G. J., Deleury, E., Perfus-Barbeoch, L., Anthouard, V., Artiguenave, F., Blok, V. C., Caillaud, M. C., Coutinho, P. M., Dasilva, C., De Luca, F., Deau, F., Esquibet, M., Flutre, T., Goldstone, J. V., Hamamouch, N., ... Wincker, P. (2008). Genome sequence of the metazoan plant-parasitic nematode Meloidogyne incognita. Nature Biotechnology, 26(8), 909-915. https://doi.org/10.1038/nbt.1482

Almeida, T. S. \& Andrade, E. P. (2010). Comunidade de nematoides em sistemas de manejo do solo. In: IIV Congresso Estadual de Iniciação Científica do IF Goiano (pp. 1-2). IIV Congresso Estadual de Iniciação Científica do IF Goiano. http://abequa.org.br/trabalhos/micropaleontologia_14.pd $\mathrm{f}$

Altieri, M. A. (1995). Agroecology: the Science of Sustainable Agriculture. CRC Press. http://hdl.handle.net/10919/65594

Anaya, R. E. \& Lombo, I. J. (2008). Identificación de nemátodos fitoparásitos asociados al cultivo de ñame espino (Dioscórea rotundata) en el corregimiento de Mateo Pérez - Sampués, municipio del Departamento de Sucre (Tesis de Grado, Universidad de Sucre). Repositorio Unisucre http://repositorio.unisucre.edu.co/handle/001/74

Arévalo, E. (2014). Dinámica de los indicadores de calidad del suelo en el manejo de sistemas agroforestales con cacao (Tesis Doctoral). Universidad Nacional Agraria. Repositorio Institucional. http://repositorio.lamolina.edu.pe/handle/UNALM/1758

Baermann, G. (1917) Eine einfache Methode zur Auffindung von Ankylostomum (Nematoden) larven in Erdproben. Geneeskundig Tijdschrift voor Nederlandsch Indië, 57, 137.

Bernard, G. C., Egnin, M. \& Bonsi, C. (2017). The Impact of Plant-Parasitic Nematodes on Agriculture and Methods of Control. In M. Manjur Shah \& M. Mahamood (Ed.), Nematology - Concepts, Diagnosis and Control (pp. 121151). 10.5772/intechopen.68958

Bogale, M., Baniya, A. \& Digennaro, P. (2020). Nematode identification techniques and recent advances. Plants, 9(10), 1-15. https://doi.org/10.3390/plants9101260

Bongers, T. \& Bongers, M. (1998) Functional diversity of nematodes. Applied Soil Ecology, 10(3), 239-251. https://doi.org/10.1016/S0929-1393(98)00123-1

Burtnik, O. J. (2006). Yerba mate : Manual de producción. INTA 
- Agencia de Extensión Rural Santo Tomé - Corrientes, 03756, 1-52.

Campos, V. P. \& Villain, L. (2005). Nematodes parasites of coffee and cocoa. In M., Luc, R.P., Sikora \& Bridge, J. (Ed.), Plant Parasitic Nematodes in Subtropical and Tropical Agriculture (pp 529-580), CABI.

Campos, V. P. \& Silva, J. R. C. (2008). Management of Meloidogyne spp. in coffee plantations. In R.M., Souza (Ed.), Plant-parasitic Nematodes of Coffee (pp. 149-163), Springer Science Business Media B.V.

Carvalho, J., Coimbra, J. L. \& Santos, F. (2011). Diversidade de fitonematóides em áreas nativas e de agricultura no cerrado baiano. Magistra. 23(4), 262-267. https://docplayer.com.br/25958064-Diversidade-defitonematoides-em-areas-nativas-e-de-agricultura-nocerrado-baiano.html

Castellón, J. (2009). Estudio de poblaciones de fitonematodos, nematodos de vida libre, hongos endofíticos y su relación con propiedades físicas y químicas del suelo en el cultivo del plátano en Rivas - Nicaragua. (Tesis de maestría, Escuela de Posgrado-CATIE). Repositorio Institucional CATIE.

http://repositorio.bibliotecaorton.catie.ac.cr/handle/1155 4/4995

Castillo, P., Vovlas, N., Troccoli, A., Liébanas, G., Palomares Rius, J. E. \& Landa, B. B. (2009). A new root-knot nematode, Meloidogyne silvestris $\mathrm{n}$. sp. (nematoda: Meloidogynidae), parasitizing european holly in northern Spain. Plant Pathology, 58(3), 606-619. https://doi.org/10.1111/j.1365-3059.2008.01991.x

Coyne, D. L., Nicol, J. M. \& Claudius-Cole, B. (2014). Practical plant nematology: A field and laboratory guide Integrated Pest Management.

Cubas Báez, A. P., Monges Zalazar, E.F. \& Quevedo Fernández, M. L. (2018). Evaluación económica de parcelas de producción de yerba mate (Ilex paraguariensis A. St-Hil) en sistemas agroforestales en el departamento de Itapúa, Paraguay. [Presentación en Jornada]. XXVI Jornadas de Jóvenes Investigadores AUGM. https://bdigital.uncu.edu.ar/fichas.php?idobjeto=13297

Derouard, L. \& Lavelle, P. (1994). Variation de la macrofaune du sol au cours des différentes étapes de la jachère dans les systèmes agricoles au Sénégal. In C., Floret (Ed.), Raccourcissement du temps de jachère, biodiversité et développement durable en Afrique Centrale (Cameroun) et en Afrique de l'Ouest (Sénégal, Mali).(pp. 47-60). Commission des Communautés Européennes.

Dinardo-miranda, L. L. (2005). Nematóides e pragas de solo em cana-de-açúcar. Encarte do Informações Agronômicas, $110,25-32$.

Edwards, C. A. \& Lofty, J. R. (1969). The influence of agricultural practice on soil micro-arthropod populations. In L.G., Sheals (Ed.), The Soil Ecosystem (pp. 234-247). Systematics Association.

Ekschmitt, K., Bakonyi, G., Bongers, M., Bongers, T., Boström, S., Dogan, H., Harrison, A., Nagy, P., O'Donnell, A. G., Papatheodorou, E. M., Sohlenius, B., Stamou, G. P. \& Wolters, V. (2001). Nematode community structure as indicator of soil functioning in European grassland soils. European Journal of Soil Biology, 37(4), 263-268. https://doi.org/10.1016/S1164-5563(01)01095-0

Enciso, V. (2019). Compendio gráfico de exportaciones agroalimentarias del Paraguay. FCA-UNA. http://www.agr.una.py/ecorural/otras_publicaciones/com pendio_grafico_exportaciones.pdf

Eno, C. F. \& Blue, W. G. (1954). The effect of anhydrous ammonia on nitrification and the microbiological population in sandy soils. Soil Science Society of America Proceedings, 18, 178-181.

Fernández, M. \& Ortega, J. (1982). Comportamiento de las poblaciones de nematodos fitoparásitos en plátano enano Cavendish. Ciencias de la Agricultura. 13, 7-17.

Ferreira, R. V., Otoboni, C. E. de M., Cruz, M. C. \& Goulart, S. L. (2007). Ocorrência de nematóides nas culturas da aceroleira, goiabeira e pessegueiro. Revista Cientifica Eletrônica de Agronomia, 7(12), 1-7. http://faef.revista.inf.br/imagens_arquivos/arquivos_dest aque/ceeoXgTr3L2dT3F_2013-5-3-15-1-43.pdf

Figueira, A. F., Berbara, R. L. L. \& Pimentel, J. P. (2011). estrutura da população de nematoides do solo em uma unidade de produção agroecológica no Estado do Rio de Janeiro, Brasil. Acta Scientiarum - Agronomy, 33(2), 223 229. https://doi.org/10.4025/actasciagron.v33i2.4716

Goulart, A. M. (2007). Diversidade de nematóides em agroecossistemas e ecossistemas naturais. Embrapa Cerrados, Documentos, 191(1), 1-71. https://www.infoteca.cnptia.embrapa.br/bitstream/doc/57 1966/1/doc191.pdf

Grigoletti Junior, A. \& Auer, C. G. (1996). Doenças da ervamate: identificação e controle. Embrapa-CNPF, Circular técnica, $25, \quad 18$ http://ainfo.cnptia.embrapa.br/digital/bitstream/item/169 66/1/circ-tec25.pdf

Grigoletti Junior, A., Auer, C. G. \& Iede, E. T. (2000). Manual de identificação de pragas e doenças da erva-mate (Ilex paraguariensis) St. Hil. Embrapa Florestas, Documentos, 44, 1-23. http://ainfo.cnptia.embrapa.br/digital/bitstream/item/160 800/1/Doc-44.pdf

Horst, R. K. (2013). Field manual of diseases on trees and shrubs. Springer. https://doi.org/10.5860/choice.51-1234

Huang, S. P. \& Cares, J. E. (2006). Nematode communities in soils under different land use systems in Brazilian Amazon and Savannah vegetation. In: F. M. S., Moreira, J. O., Siqueira \& L., Brussaard (Ed.). Soil biodiversity in Amazonian and other Brazilian ecosystems. (pp. 163 183). 10.1079/9781845930325.0163

IICA, COSAVE \& PHSAIC. (2019). Metodologia de avaliação de impacto socioeconômico de medidas fitossanitárias $e$ Guia de aplicação. https://repositorio.iica.int/bitstream/handle/11324/7908/ BVE19040211p.pdf?sequence $=3$ \&isAllowed $=\mathrm{y}$

Jenkins, W. R. (1964). A rapid centrifugal-flotation technique for separating nematodes from soil. Plant Disease Reporter, $\quad 48(9), \quad 692$. https://www.cabdirect.org/cabdirect/abstract/196508011 05

Jones, J. T., Haegeman, A., Danchin, E. G. J., Gaur, H. S., Helder, J., Jones, M. G. K., Kikuchi, T., ManzanillaLópez, R., Palomares-Rius, J. E., Wesemael, W. M. L. \& Perry, R. N. (2013). Top 10 plant-parasitic nematodes in molecular plant pathology. Molecular Plant Pathology, 14(9), 946-961. https://doi.org/10.1111/mpp.12057

Kandji, S. T., Ogol, C. K. P. O. \& Albrecht, A. (2001). Diversity of plant-parasitic nematodes and their relationships with some soil physico-chemical characteristics in improved fallows in western Kenya. Applied Soil Ecology, 18(2), 143-157. https://doi.org/10.1016/S0929-1393(01)001573

Khan, Z. \& Kim, Y. H. (2007). A review on the role of predatory soil nematodes in the biological control of plant parasitic nematodes. Applied Soil Ecology, 35(2), 370-379. https://doi.org/10.1016/j.apsoil.2006.07.007

Kimenju, J. W., Odero, G. O. M., Mutitu, E. W., Wachira, P. M., 
Narla, R. D. \& Muiru, W. M. (2009). Suitability of locally available substrates for Oyster Mushroom (Pleurotus ostreatus) cultivation in Kenya. Asian Journal of Plant Sciences, $\quad 8(7), \quad 510-514$. https://doi.org/10.3923/ajps.2009.510.514

Kirsch, V. G., Kulczynski, S. M., Gomes, C. B., Bisognin, A., Gabriel, M., Bellé, C. \& Lima-Medina, I. (2016). Helicotylenchus Associadas À Soja No Rio Grande Do Sul. Nematropica, 46(2), 197-208. https://journals.flvc.org/nematropica/article/view/90812

Lozano, L. A. \& Santos, B. dos. (1991). Ocorrência de nematoides do gênero Meloidogyne em mudas de ervamate no estado do Paraná. Revista de Agricultura, 66(2), 203-205.

https://www.revistadeagricultura.org.br/index.php/revist adeagricultura/article/view/903

Luc, M., Sikora, R. \& Bridge, J. (2005). Plant Parasitic nematodes in subtropical and tropical agriculture. CABI. https://www.researchgate.net/publication/236985095_Pl ant_Parasitic_Nematodes_in_Subtropical_and_Tropical_ Agriculture_2nd_Edition

Maciel, A., Villalba, J., Benítez, M. \& Vera, M. R. (2020). Síntesis Estadísticas: Año Agrícola 2019/2020. Dirección de Censos y Estadísticas Agropecuarias [DCEA]Ministerio de Agricultura y Ganadería [MAG]. 1-50.

Magurran, A. E. (2004). Measuring biological diversity. Blackwell. http://www.bionica.info/Biblioteca/Magurran2004MeasuringBiological. pdf

Meneses, A., Pocasangre, L. E., Somarriba, E., Riveros, A. S. \& Rosales, F. E. (2003). Diversidad de hongos endofíticos y abundancia de nemátodos en plantaciones de banano y plátano de la parte baja de los territorios indígenas de Talamanca. Agroforestería de las Américas, 10(37-38), 59-62. http://hdl.handle.net/11554/6077

Mesa-Valle, C. M., Garrido-Cardenas, J. A., Cebrian-Carmona, J., Talavera, M. \& Manzano-Agugliaro, F. (2020). Global Research on Plant Nematodes. Agronomy, 10(8), 1148. https://doi.org/10.3390/agronomy10081148

Neher, D. A. (1999). Nematode communities in organically and conventionally managed agricultural soils. Journal of Nematology, $\quad 31(2), \quad 142-154$. https://www.ncbi.nlm.nih.gov/pmc/articles/PMC262036 $8 /$

Neher, D. A. \& Darby, B. J. (2009). General community indices that can be used for analysis of nematode assemblages. In M.J., Wilson, \& T., Kakouli-Duarte (Ed.), Nematodes as environmental indicators (pp. 107-123). CAB International.

https://www.researchgate.net/profile/Deborah-

Neher/publication/250612802_General_community_indi ces_that_can_be_used_for_analysis_of_nematode_asse mblages/links/0c96051ec373c27c33000000/Generalcommunity-indices-that-can-be-used-for-analysis-ofnematode-assemblages.pdf

Neher, D. A., Nishanthan, T., Grabau, Z. J. \& Chen, S. Y. (2019). Crop rotation and tillage affect nematode communities more than biocides in monoculture soybean. Applied Soil Ecology, 140(1), 89-97. https://doi.org/10.1016/j.apsoil.2019.03.016

Neves, W. S., Dias, M. S. C. \& Barbosa, J. G. (2009). Flutuação populacional de nematoides em bananais de Minas Gerais e Bahia (anos 2003 a 2008). Nematologia Brasileira, 33(4), 281-285.

Norton, D. C. (1978). Ecology of Plant-parasitic Nematodes. Wiley Interscience.

Oliveira, Y. M. de. \& Rotta, E. (1985). Área de distribuição natural de erva-mate (Ilex paraguariensis St. Hil.). Embrapa CNPF, 15, 17-36. http://ainfo.cnptia.embrapa.br/digital/bitstream/item/102 798/1/AreaDistribuicao.pdf

Ovando, C. E. (2014). Biodiversidad de comunidades de nematodos asociados a café (Coffea arabica L.), diagnóstico y servicios desarrollados en Finca las Nubes, San Francisco Zapotitlán, Suchitepéquez, Guatemala, C.A. (Tesis de grado, Universidad San Carlos de Guatemala). Repositorio USAC. http://www.repositorio.usac.edu.gt/2753/

Palomares-Rius, J. E., Castillo, P., Montes-Borrego, M., Müller, H. \& Landa, B. B. (2012). Nematode community populations in the rhizosphere of cultivated olive differs according to the plant genotype. Soil Biology and Biochemistry, $45(1), \quad 168-171$. https://doi.org/10.1016/j.soilbio.2011.11.009

Paoletti, M. G., Favretto, M. R., Marchiorato, A., Bressan, M. \& Babetto, M. M. (1993). Biodiversità in pescheti forlivesi. En M.G., Paoletti (Ed.), Biodiversità negli Agroecosistemi (pp. 20-56). Osservatorio Agroambientale.

Peraza, W. (2010). Nematofauna asociada al cultivo de café (Coffea arabica) y orgánico y convencional en Aserrí, Costa Rica. Ingenierías \& Amazonia, 3(2), 105-112. https://www.uniamazonia.edu.co/revistas/index.php/inge nierias-y-amazonia/article/view/74/0

Ramos, R., Kaspary, T. E., Balardin, R. R., Nora, D. D., Antonioli, Z. I. \& Bellé, C. (2019). Plantas daninhas como hospedeiras dos nematoides-das-galhas. Revista Agronomia Brasileira, 3(3), 1-3. https://doi.org/10.29372/rab201906

Ritzinger, C. H., Fancelli, M. \& Ritzinger, R. (2010). Nematoides: Bioindicadores de sustentabilidade e mudanças edafoclimáticas. Revista Brasileira de Fruticultura, 32(4), 1289-1296. https://doi.org/10.1590/S0100-29452010000400045

Rivera, M. J., Rodriguez-Saona, C., Jennings, D. E. \& Koppenhöfer, A. M. (2015). Assessing the impact of cultivation and plant domestication of highbush blueberry (Vaccinium corymbosum) on soil properties and associated plant-parasitic nematode communities. Soil Biology and Biochemistry, 88, 25-28. https://doi.org/10.1016/j.soilbio.2015.05.010

Rocha, M., Nunes, H. \& Sardeiro, S. (2019). Quantificação e Distribuição espacial de fitonematóides em uma área hortícola no Oeste da Bahia. Magistra, 30(1), 406-412. https://magistraonline.ufrb.edu.br/index.php/magistra/art icle/view/698

Santiago, D. C., Krzyzanowski, A. A. \& Homechin, M. (2000). Behavior of Ilex paraguariensis St. Hilaire, 1822 to Meloidogyne incognita and M. paranaensis and their influence on development of plantlets. Brazilian Archives of Biology and Technology, 43(2), 139-142. https://doi.org/10.1590/S1516-89132000000200001

Semensatto Junior, D.L. (2003). Aplicação de índices de diversidade em estudos envolvendo associações entre foraminíferos e tecamebas recentes: uma breve discussão. Em: Anais do IX Congresso da associação brasileira de estudos do quaternário (pp. 1-5). IX Congresso da associação brasileira de estudos do quaternário. http://abequa.org.br/trabalhos/micropaleontologia_14.pd $\mathrm{f}$

Shannon, C. E. \& Weaver, W. (1963). The mathematical theory of information. Urbana. https://monoskop.org/images/b/be/Shannon_Claude_E_ Weaver_Warren_The_Mathematical_Theory_of_Comm 
unication_1963.pdf

Simpson, E. H. (1949). Measurements of diversity. Nature, 163, 688. https://doi.org/10.1038/163688a0

Suárez, Z. \& Rosales, L. (2004). Problemas nematológicos en musáceas. Revista Digital CENIAP HOY, 6. http://www.ceniap.gov.ve/ceniaphoy3/articulos/n6/arti/ suarez_z/arti/suarez_z.htm

Tomazini, M. D., Ferraz, L. C. C. B. \& Monteiro, A. R. (2008). Abundância e diversidade de nematóides em área contíguas de vegetação natural e submetidas a diferentes tipos de uso agrícola. Nematologia Brasileira, 32(3), 185-193.

https://www.researchgate.net/publication/242424289_A bundancia_e_Diversidade_de_Nematoides_em_Areas_C ontiguas_de_Vegetacao_Natural_e_Submetidas_a_Difer entes_Tipos_de_Uso_Agricola
Umesh, K. C., Ferris, H. \& Bayer, D. E. (1994). Competition between the plant-parasitic nematodes Pratylenchus neglectus and Meloidogyne chitwoodi. Journal of Nematology, 26(3), 286-295. https://www.ncbi.nlm.nih.gov/pmc/articles/PMC261950 $0 /$

Uramoto, K., Walder, J. M. M. \& Zucchi, R. A. (2005). Análise quantitativa e distribuição de populações de espécies de Anastrepha (Diptera: Tephritidae) no campus Luiz de Queiroz, Piracicaba, SP. Neotropical Entomology, 34(1), 33-39. 566X2005000100005

Wardle, D. A. \& Yeates, G. W. (1993). The dual importance of competition and predation as regulatory forces in terrestrial ecosystems: evidence from decomposer foodwebs. Oecologia, 93(2), 303-306. https://doi.org/10.1007/BF00317685 